\title{
Simultaneous Radio and X-Ray observations of Crab Pulsar
}

\author{
A. K. Basu ${ }^{1}$, B. C. Joshi ${ }^{1}$ and D. Bhattacharya ${ }^{2}$ \\ ${ }^{1}$ National Centre for Radio Astrophysics, Tata Institute of Fundamental Research, \\ Pune University Campus, Pune 411007 \\ email: avishek@ncra.tifr.res.in \\ email: bcj@ncra.tifr.res.in \\ ${ }^{2}$ Inter-University Centre for Astronomy and Astrophysics, \\ Pune University Campus, Pune 411007 \\ email: dipankar@iucaa.in
}

\begin{abstract}
Crab Pulsar (PSR B0531+21) is known to emit pulsed emission in all bands of the electromagnetic spectrum. It also emits giant radio pulses (GRPs) frequently, which are roughly a hundred to million times brighter than the normal pulses. We aim to study whether there is a significant X-ray enhancement correlated with the occurrence of GRPs, using simultaneous observations with the ASTROSAT, the Giant Meterwave Radio telescope (1300 MHz) and the Ooty Radio telescope (325 MHz). This required determination of fixed pipeline offsets between different instruments. We find the offset between ASTROSAT and GMRT to be $-30.181 \pm 0.095$ $\mathrm{ms}$ and that between ASTROSAT and ORT to be $-18.4 \pm 0.2 \mathrm{~ms}$. Our preliminary results with $1300 \mathrm{MHz}$ data also show a break in pulse intensity distribution at $\sim 33$ Jy in the main pulse and $\sim 28$ Jy in the inter-pulse.
\end{abstract}

Keywords. instrumentation: interferometers, line: profiles, Pulsar PSR J0531+2200, telescopes

\section{Introduction}

The Crab pulsar (PSR B0531+21), residing in the Crab nebula, emits pulsed emission across the electromagnetic spectrum. Its profile has two distinct peaks at $1400 \mathrm{MHz}$, with the brighter component called the Main Pulse (MP) and the other the Inter-pulse (IP). Both these components are also seen at X-ray band, with an additional bridge emission between them. The radio pulse profile is offset from the X-ray pulse profile by $340 \mu s$ (Rots et al. 2004). In addition to average emission, this pulsar also shows intense narrow pulses, sometimes a million times more than the average pulse energy, at radio waveband. The emission mechanism responsible for these "giant radio pulses (GRPs)" is not yet understood. Only a handful of pulsars, other than the Crab pulsar, are known to emit GPs (Joshi et al. 2004). In these pulsars, GRPs in radio appear to be in phase with the X-ray emission (Johnston, S. et al. 2004). We wish to investigate whether there is a significant enhancement in the photon counts at the time of GRPs in the Crab pulsar, which can help in constraining the high energy emission location as well as the emission mechanism for these pulses. The high time resolution capability (10 to $20 \mu s$ ) available with instruments aboard India's first multi-waveband astronomical satellite, ASTROSAT, promises to be very useful for this study with simultaneous observations with ground based radio telescopes, such as the Giant Meterwave Radio Telescope (GMRT) and the Ooty Radio Telescope (ORT). However, this requires calibrating the timing offset between the different instruments. Here, the results of such calibration observations are presented. 


\section{Instruments, Observations and Data}

ASTROSAT (Agrawal 2017) was launched on 25th September 2015. It has five different instruments on-board - Cadmium Zinc Telluride Imager (CZTI), Large Area X-ray Proportional Counter (LAXPC), Soft X-ray Telescope (SXT), Ultra-Violet Imaging Telescope (UVIT) and Scanning Sky Monitor (SSM). It is a unique facility, which covers the electromagnetic spectrum from 3000 angstroms to $280 \mathrm{KeV}$. For this work, Crab pulsar was simultaneously observed with the CZTI, the GMRT and the ORT over multiple epochs. The GMRT is an array of 30 telescopes, each of 45-m diameter, whereas the ORT is a $530-\mathrm{m}$ long and $30-\mathrm{m}$ wide cylindrical paraboloid telescope. Since the ASTROSAT mission launch, the Crab pulsar was monitored by the ORT at $326.5 \mathrm{MHz}$ almost every day, which has enabled us to obtain a very precise timing solution. Fifteen antennas of the GMRT with short baselines were combined in a phased array mode to produce an effective single dish antenna of radius $\sim 1.8 \mathrm{kms}$ at $1300 \mathrm{MHz}$. At both the telescopes, data were recorded as raw voltages over $16 \mathrm{MHz}$ band-pass with $30 \mathrm{~ns}$ sampling time. These were then coherently dedispersed to produce a time series, which has been folded using the ephemeris of the pulsar, obtained from our timing solution. Then, the Time of Arrivals (ToAs) were calculated using Taylor's method (Taylor, J. H. 1992 ). Crab Pulsar is known for its timing noise, which is manifested in the wandering of ToA residuals. We removed the timing noise using FITWAVES of Tempo2 (Hobbs, G. et al. 2006). The other source of wandering of ToAs is the variations in dispersion measure (DM). We used the DM values provided by Jodrell bank from their monthly Crab ephemeris and interpolate them to get the epoch dependent DM estimates. We further improved the DM values by using the simultaneous observations of with the GMRT and the ORT.

\section{Results and Discussion}

Residuals from the phase connected solution of ToA-s, at different wavebands, were whitened using the methods mentioned in Sec 2. The residuals, thus obtained, lie on parallel tracks for different telescopes, separated by the fixed offsets in each data recording pipeline. These offsets were fit using tempo2. We find that the offset between ASTROSAT CZTI and GMRT is $-30.181 \pm 0.095 \mathrm{~ms}$ and that between ASTROSAT CZTI and ORT is $-18.4 \pm 0.2 \mathrm{~ms}$. As the GMRT is an interferometer, it can resolve the pulsar from the nebula. The background noise level $(\sigma)$ for GMRT observations is $\sim 2.4 \mathrm{Jy}$, an order of magnitude lower than previous radio observations, primarily due to lower contributions from the bright Crab nebula (nebular flux density $\sim 931 \mathrm{Jy}$ at L-band). This helps to detect even the fainter pulses. We discovered a break in the single pulse intensity distribution at $1300 \mathrm{MHz}$, which we believe separates GRPs from normal pulses. From our preliminary analysis, we find the cut-off intensity above which we will call the pulses to be GRPs is $\sim 33 \mathrm{Jy}$ in MP window and $\sim 28 \mathrm{Jy}$ in IP window.

\section{References}

Agrawal, P. C. 2017, Journal of Astrophysics and Astronomy, 38, 27

Hobbs, G., Edwards, R., \& Manchester, R. 2006, MNRAS, 369, 655

Johnston, S. 2004, in: F. Camilo \& B. M. Gaensler (eds.), Young Neutron Stars and Their Environments, Proc. IAU Symposium No. 218 (San Francisco: ASP), p. 1

Joshi, B. C., Kramer, M., Lyne, A. G., McLaughlin, M. A., \& Stairs, I. H. 2004, Proc. IAU Symp. 218, 319

Rots, A. H., Jahoda, K., \& Lyne, A. G. 2004, ApJ(Letters), 605, L129

Taylor, J. H. 1992, Philosophical Transactions: Physical Sciences and Engineering, 117 